\title{
Spontaneous Hyphema from Iris Microhemangiomatosis in an Elderly Patient with Hypertensive Crisis
}

\author{
Pedro J. Nuova ${ }^{a}$ Dinah Zur $^{\mathrm{b}} \quad$ Anat Loewenstein $^{\mathrm{b}}$ Matias Iglicki $^{\mathrm{c}}$ \\ aPrivate Retina Office, Tucuman, Argentina; ${ }^{b}$ Ophthalmology Division, Tel Aviv Medical \\ Center affiliated to the Sackler Faculty of Medicine, Tel Aviv University, Tel Aviv, Israel; \\ 'Private Retina Office, University of Buenos Aires, Buenos Aires, Argentina
}

\section{Keywords}

Iris microhemangiomatosis $\cdot$ Hyphema $\cdot$ High blood pressure $\cdot$ Fluorescein angiography

\begin{abstract}
Background: Iris microhemangiomatosis is a rare vascular iris tumor, with potential severe complications such as increased intraocular pressure (IOP). We aim to describe a case report of a patient presenting with hyphema secondary to iris microhemangiomatosis triggered by excessive high blood pressure. Case Presentation: A 74-year-old woman was treated for hypertensive crisis. After her high blood pressure had been controlled and stabilized, she was discharged home. However, the same day, she complained about an acute decrease in vision in her left eye. Best corrected visual acuity was 20/20 on the right eye and 20/200 on the left eye. On biomicroscopy, a hyphema was seen. Iris neovascularization was absent, IOP and fundus examination were normal. After spontaneous resolution of the hyphema, a fluorescein angiography of the anterior segment was performed, which revealed bilateral subtle early hyperfluorescence with late staining scattered at the pupillary margin. The patient was diagnosed with iris microhemangiomatosis. During the follow-up of 24 months, the blood pressure was stable and well controlled. The patient did not experience any recurrent hemorrhage. Discussion and Conclusion: Spontaneous hyphema is the most common complication of iris vascular tumors. We report the occurrence of a spontaneous hyphema triggered by uncontrolled blood pressure in a patient with a very rare condition, i.e., iris microhemangiomatosis. In order to
\end{abstract}


avoid complications of microhemangiomatosis such as uncontrolled glaucoma or recurrent bleeding requiring surgery, blood pressure should be monitored closely and controlled.

(C) 2020 The Author(s)

Published by S. Karger AG, Basel

\section{Background}

Tumors of the iris have a relatively low incidence, and vascular tumors of the iris are even more uncommon, comprising $2 \%$ of all iris tumors. Shields et al. [1] recently published the largest series of 3,680 cases of iris tumors. Only 57 eyes presented vascular iris tumors, including racemose hemangioma, cavernous hemangioma, capillary hemangioma, varix, and microhemangiomatosis.

Iris microhemangiomatosis is usually characterized by bilateral small clusters of tightly coiled blood vessels at the pupillary margin [1-3]. Iris microhemangiomatosis is typically found in elderly adults. The etiology seems to be idiopathic.

We aim to describe a case report of an uncommon trigger for hyphema in an elderly patient with iris microhemangiomatosis.

\section{Case Presentation}

A 74-year-old woman was complaining about chest pain and shortness of breath. At the emergency room she was diagnosed with hypertensive crisis and blood pressure of 280/140 was measured. After adjusting the hypertensive medication, she was discharged home.

Shortly after her discharge, she presented to our eye institute complaining of acute decreased vision in her left eye. Prior ocular history was negative. She reported that her vision had dropped immediately after getting up that day. On her first visit to our institution, her best corrected visual acuity (BCVA) was 20/20 on her right eye, and 20/200 on her left eye. Biomicroscopy examination revealed a dense hyphema coming from active bleeding (Fig. 1a, b), without evidence of neovascularization of the iris or the angle on gonioscopy. Moreover, a small iris nevus was seen in the upper iris. The blood was clearly coming from the pupillary border. Examination of the right eye was normal. Intraocular pressure (IOP) was $15 \mathrm{~mm} \mathrm{Hg}$ in both eyes. Fundus examination showed no abnorm. Macular optical coherence tomography (OCT) (Cirrus HD-OCT, Zeiss, Germany) was normal. Fundus fluorescein angiography (FA) showed no retinal vascular alterations. As the IOP was normal, the patient was discharged with topical treatment of prednisolone acetate 1\% (PredForte) and cyclopentolate 1\% TID and scheduled for follow-up.

The patient was seen in our clinic 15 days later and the hyphema had resolved. An iris FA was performed showing subtle early hyperfluorescence with late staining scattered at the pupillary margin bilaterally presenting ectatic dilatation of the iris vessels (Fig. 2a, b, 3a, b). The patient was diagnosed with iris microhemangiomatosis.

The patient has been followed for over 24 months without recurrence of the hyphema and she had a BCVA of 20/20 at distance in the affected eye. Blood pressure was well controlled during the follow-up. 


\section{Discussion and Conclusion}

Spontaneous hyphema is the most common complication of iris vascular tumors. Iris vascular lesions represent $2 \%$ of all iris tumors, and microhemangiomatosis is a very uncommon entity ( $5 \%$ of all vascular iris tumors) [1]. The small vascular clusters at the pupillary border carry the risk for spontaneous hyphema and secondary increase in IOP $[2,4,5]$.

We hypothesize that the hypertensive crisis in the patient presented here triggered the occurrence of the hyphema. Notably, all cases of iris microhemangiomatosis were diagnosed in patients older than 60 years old. Therefore, we strongly believe that measurement of blood pressure should be performed in all patients presenting with spontaneous hyphema. As this diagnosis is difficult to establish on clinical examination without the use of iris FA, this condition might be underdiagnosed.

The differential diagnosis of spontaneous hyphema includes iris neovascularization, iris or ciliary body neoplasms, uveitis-glaucoma-hyphema syndrome, and blood dyscrasias [6]. While rubeosis presents with vessel leakage on FA, iris microhemangiomatosis shows dilated ectatic vessels without any leakage.

Another important tool in the workup of patients with spontaneous hyphema is ultrasound biomicroscopy in order to rule out neoplasms of the iris and ciliary body. In the current case, ultrasound biomicroscopy was not performed as the cause of the hyphema was demonstrated on FA.

Uncontrolled high blood pressure might produce hyphema due to iris microhemangiomatosis. Even more, in this case report, bleeding occurred only once. However, it has been published that spontaneous hyphema from iris microhemangiomatosis can be recurrent and treatment should be performed. This led us to the conclusion that in order to avoid complications of microhemangiomatosis such as uncontrolled glaucoma or recurrent bleeding requiring surgery etc., blood pressure should be controlled.

\section{Statement of Ethics}

There was no need for ethics approval or consent.

\section{Disclosure Statement}

The authors declare that they have no competing interests.

\section{Funding Sources}

No funding or grant support.

\section{Author Contributions}

P.J.N., D.Z., A.L., and M.I. were involved in data analysis, writing and reviewing the paper. P.J.N. was also involved in data acquisition. All authors read and approved the final manuscript. 


\section{Case Reports in Ophthalmology}

\section{References}

1 Shields CL, Kancherla S, Patel J, Vijayvargiya P, Suriano MM, Kolbus E, et al. Clinical survey of 3680 iris tumors based on patient age at presentation. Ophthalmology. 2012 Feb;119(2):407-14.

2 Malik K, Welch RJ, Shields CL. Spontaneous Hyphema in a Middle-aged Woman. JAMA Ophthalmol. 2018 Jul;136(7):829-30.

3 Winnick M, Margalit E, Schachat AP, Stark WJ. Treatment of vascular tufts at the pupillary margin before cataract surgery. Br J Ophthalmol. 2003 Jul;87(7):920-1.

4 Ni N, Johnson TV, Koval MS, Shields CL. Iris microhemangiomatosis with videographically documented active bleeding and vision loss. JAMA Ophthalmol. 2013 Dec;131(12):1649-51.

5 Shields JA, Bianciotto C, Kligman BE, Shields CL. Vascular tumors of the iris in 45 patients: the 2009 Helen Keller Lecture. Arch Ophthalmol. 2010 Sep;128(9):1107-13.

6 Terry JE, Roberts DK. Differential diagnosis of spontaneous hyphema associated with central retinal vein occlusion. J Am Optom Assoc. 1986 Sep;57(9):688-92.

Pedro J. Nuova and Dinah Zur contributed equally to this work.
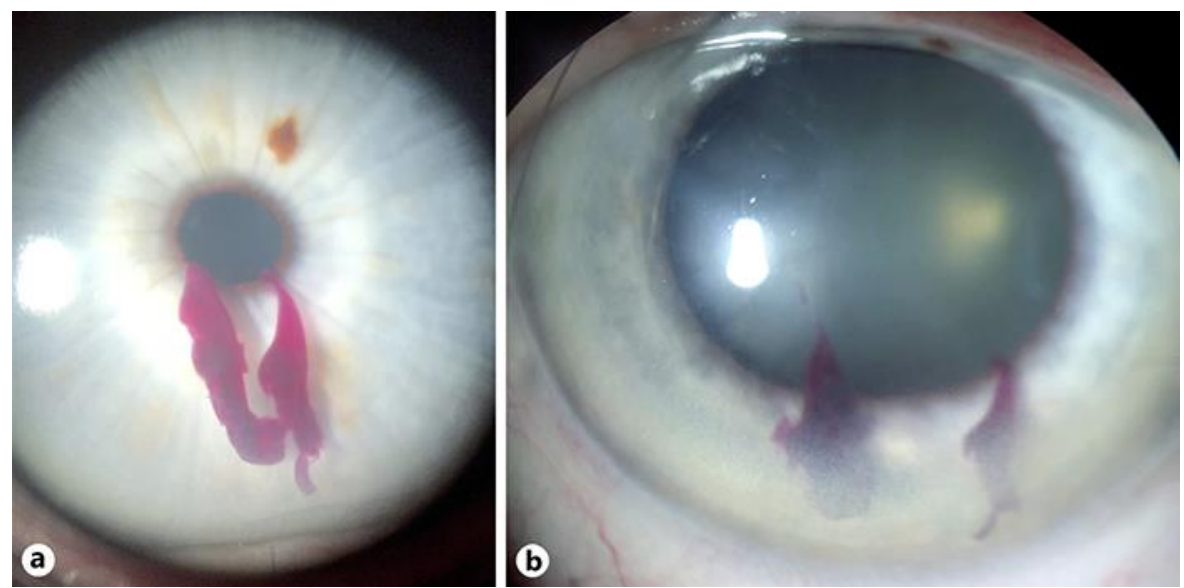

Fig. 1. Anterior segment photograph on initial presentation. a Hyphema in the left eye originating from the pupillary border. An iris nevus can be seen at 1 o'clock. b After pupillary dilation, the hyphema can still be seen connected to the pupillary border. 


\section{Case Reports in Ophthalmology}
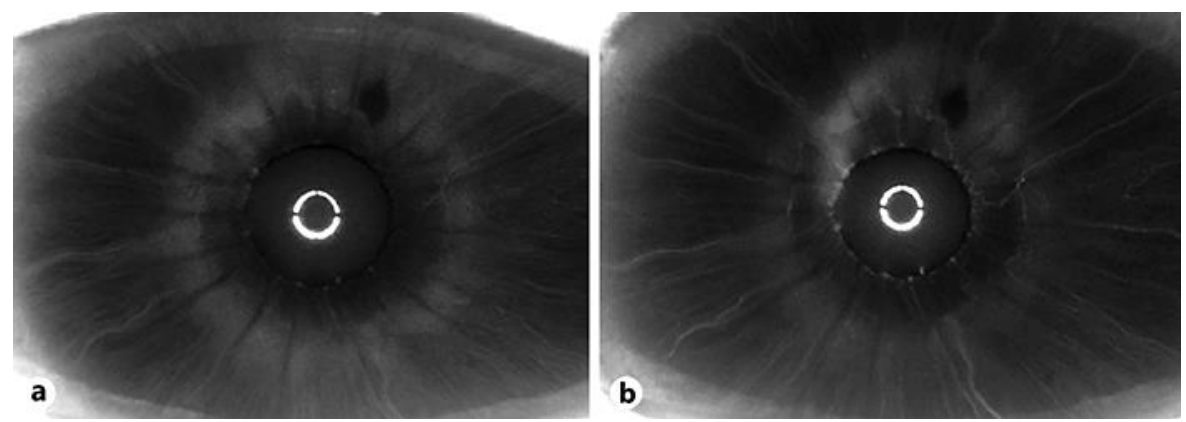

Fig. 2. Anterior segment fluorescein angiography of the left eye. Anterior segment fluorescein angiography demonstrates several tiny, pinpoint foci of hyperfluorescence (a) with leakage at the iris pupillary margin in the late phase (b). At 1 o'clock, the iris nevus presents as a hypofluorescent lesion.
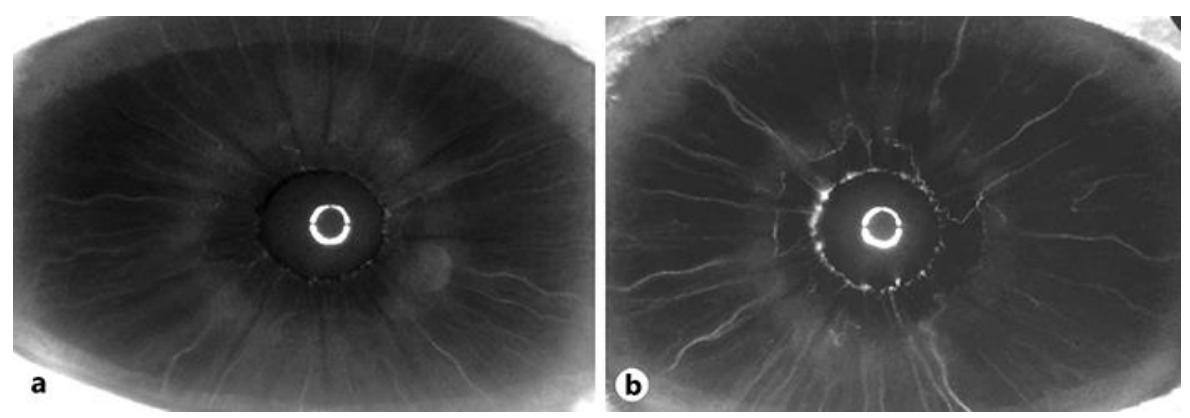

Fig. 3. Anterior segment fluorescein angiography of the right eye. Fluorescein angiography showing small vascular tufts scattered at the pupillary margin (a), showing late leakage (b). 